\title{
Evolución terapéutica en paciente sometido a cirugía cardiotorácica
}

\author{
CARla PitARCh Albado \\ cpitarch12@gmail.com \\ ISABEL PILAR ROS CARDO \\ isabelrc18@gmail.com \\ Paula Recacha Ponce \\ paularecacha@hotmail.com \\ AARÓN RIBES PORCAR \\ aaronribesporcar@gmail.com \\ María Desamparados Bernat Adell \\ bernatm@uji.es
}

\section{Resumen}

Introducción: Paciente que presenta una patología pulmonar, neoplasia del lóbulo inferior derecho, sometido a cirugía de lobectomía inferior derecha más linfadenectomía. Método: El trabajo se fundamenta en la aplicación del modelo de Virginia Henderson, basado en la clasificación de las 14 necesidades según el grado de dependencia e independencia, con el fin de llevar a cabo la valoración del paciente. Mediante los datos recogidos en esta valoración se identifican los problemas reales y potenciales del paciente, que ayudan en la elaboración de los diagnósticos de enfermería (NANDA). Una vez establecido el diagnóstico, se plantean unos NOC y NIC. Resultados: Mediante monitorización respiratoria de la saturación de $0_{2}$ y la frecuencia respiratoria, una media de 3 o 4 veces por día, y basándonos en la observación del adecuado uso de la musculatura accesoria el paciente, el día del alta clínica había superado el patrón respiratorio ineficaz. La inserción de drenajes torácicos, de catéter venoso periférico y la herida quirúrgica resultado de la intervención, motivaban un riesgo de infección, al alta el riesgo de infección queda suprimido. Se evidencia un riesgo moderado de caídas, una dificultad en la realización independiente de los autocuidados relacionados con la higiene, con vestir/desvestirse y con trasferencia para el uso del inodoro, ya que necesitaba ayuda parcial y supervisión. El riesgo de caídas se controló y volvió a ser autónomo en autocuidados. Conclusiones: Identificar precozmente las necesidades del paciente permitió controlarlas y evitar riesgos.

Palabras clave: cirugía, enfermería, cuidados, tórax. 


\begin{abstract}
Introduction: Patient with a pulmonary pathology, neoplasm of the right lower lobe, had surgery right lower lobectomy plus lymphadenectomy. Method: The work is based on applying the model of Virginia Henderson, based on the classification of the 14 requirements to the degree of dependence and independence, in order to carry out the evaluation of the patient. Using data collected in this assessment the patient's actual and potential problems, which help in the development of nursing diagnoses (NANDA) are identified. Once the diagnosis is established, a NOC and NIC arise. Results: By respiratory monitoring $\mathrm{O}_{2}$ saturation and respiratory rate, an average of 3 or 4 times per day, and based on observation of the proper use of the accessory muscles the patient, the discharge day clinic had exceeded the ineffective breathing pattern. The insertion of chest tubes, peripheral venous catheter and the surgical result of the intervention, motivated a risk of infection, high risk of infection is suppressed. A moderate risk of falling, a difficulty in conducting independent self-care related to hygiene, to dress / undress and transfer to use the toilet is evidence, as needed partial care and supervision. The risk of falling is controlled and returned to be independent in self-care. Conclusions: Early identification of patients' needs allowed to control and avoid risks.
\end{abstract}

Keywords: surgery, nursing, care, thorax.

\title{
Introducción
}

Hombre de 62 años residente en Coimbra, que ingresó en el servicio de Cirugía Cardiotorácica del Hospital Universidad de Coimbra el día 13/01/2014.

El ingreso fue programado derivado a través del Servicio de Neumología del mismo hospital para ser sometido a cirugía (lobectomía inferior derecha más linfadenectomía el día 14/01/2014).

El paciente está casado y tiene una hija de 29 años, la cual no tiene pareja. Vive con ellas y tiene muy buena relación, de hecho ellas todos los días a la hora de las visitas están el hospital y pasan toda la tarde con él. Ninguna de ellas tiene antecedentes relevantes.

Es socialmente activo, aún ejerce de agricultor y tienen buenos lazos sociales y familiares. En cuanto a los estudios académicos, el paciente remarca tener estudios básicos obligatorios.

Este caso describe el periodo post-operatorio completo, con su respectiva evolución hasta el momento del alta clínica, de un paciente que presentaba una patología pulmonar, neoplasia del lóbulo inferior derecho, siendo sometido a cirugía de lobectomía inferior derecha más linfadenectomía. 


\section{Objetivos}

- La aplicación de los fundamentos teóricos de la enfermería a la práctica asistencial mediante la realización de las intervenciones correspondientes.

- Desarrollo de la capacidad de valoración frente a la persona como unidad biopsicosocial.

- Realización de las intervenciones de enfermería de forma individual, con la posterior evaluación en el cumplimiento de los objetivos atendiendo a las respuestas humanas dentro del proceso de salud-enfermedad.

\section{Método}

El trabajo comienza con la aplicación del modelo de las 14 necesidades de Virginia Henderson, modelo basado en la clasificación de las necesidades según el grado de dependencia e independencia, con el fin de llevar a cabo la valoración del paciente (Henderson, 2009). Mediante los datos recogidos en esta valoración se identifican los problemas reales y potenciales del paciente que ayudan en la elaboración de los diagnósticos de enfermería mediante la clasificación NANDA (North American Nursing Diagnosis Association). Una vez establecido el diagnóstico, se plantean unos objetivos NOC (Nursing Outcomes Classification) y unas intervenciones NIC (Nursing Interventions Classification) (metodología enfermera).

\section{Resultados}

El paciente ingresó en el servicio siendo autónomo en lo que respecta a las necesidades básicas de la vida diaria. Este caso presenta una breve cronología de la evolución del paciente en los días posteriores a la cirugía hasta el alta hospitalaria, recalcando el cumplimiento de los objetivos establecidos tras aplicación del modelo de Virginia Henderson, ver anexo 1.

El usuario durante el periodo postoperatorio (15/1/14,16/1/14,17/1/14) presentaba un patrón de respiración ineficaz en episodios diarios (1 vez por día) por presentar periodos de taquipnea, acompañada de manejo inadecuado de la musculatura accesoria. Mediante terapia respiratoria, monitorizando una media de 3 o 4 veces por día las saturaciones de $0_{2}$ y la frecuencia respiratoria, y basándonos en la observación del adecuado uso de la musculatura accesoria, el paciente el día del alta clínica (18/1/14) había superado este patrón de respiración ineficaz.

Debido a la inserción de drenajes torácicos anterior y posterior, del catéter venoso periférico en la mano derecha y de la herida quirúrgica derecha lateral-posterior-costal el paciente presentaba un riesgo de infección. El tercer día postoperatorio (17/1/14) fueron retirados los drenajes torácicos y el catéter venoso periférico, sin ninguna incidencia. En cuanto a la herida quirúrgica no presentaba ningún tipo de signo de infección (rubor, inflamación o enrojecimiento), con buen aspecto y proceso cicatricial normal. De tal modo, que el día del alta el riesgo de infección quedo suprimido.

Durante el periodo postoperatorio, el paciente debido al dolor agudo intermitente e intenso en la región derecha lateral donde tuvo lugar la incisión quirúrgica, y al tener colocados los drenajes torácicos y el sistema de suero, presentaba tanto un riesgo moderado de caídas 
como una dificultad en la realización independiente de los autocuidados para higiene personal, para vestir/desvestirse y para desplazarse al inodoro, ya que necesitaba una ayuda parcial y supervisión. El tercer día del postoperatorio, cuando se retiraron los drenajes, el sistema se suero y el dolor había mejorado tras aplicación de medidas no farmacológicas (masaje y relajación), el riesgo de caídas se abolió y volvió a ser autónomo en los autocuidados. Juntamente con esto, el paciente presentaba una baja autoestima situacional al inicio del periodo postoperatorio, motivada por la pérdida de la capacidad para realizar las actividades básicas de la vida diaria. Al finalizar su periodo de ingreso hospitalario y reincorporarse al rol social mejoró su autoestima.

\section{Discusión y conclusiones}

Durante la realización de este estudio se han alcanzado y cumplido los objetivos propuestos para el mismo, partiendo de las teorías sobre valoración e intervención fue realizado el estudio, identificados diagnósticos de enfermería y realizados las intervenciones correspondientes a los mismos.

\section{Referencias bibliográficas}

Henderson, V. (19 / marzo / 2009). «Aprende en Línea». Extraído de Plataforma académica para pregrado y posgrado. Universidad de Antioquia: http://aprendeenlinea.udea.edu. $\mathrm{co} / \mathrm{lms} / \mathrm{moodle} / \mathrm{mod} / \mathrm{resource} / \mathrm{view}$. php?inpopup=true\&id=61971.

Metodología Enfermera, (sin fecha). Extraído de Listado de Diagnósticos NANDA. Actualización en enfermería: http://enfermeriaactual.com/listado-de-diagnosticos-nanda/.

\section{Anexo 1}

Valoración según las 14 necesidades de Virginia Henderson

\begin{tabular}{|l|l|}
\hline Necesidad & Manifestaciones de dependencia \\
\hline 1. Necesidad de respirar & - Presenta episodios diarios (más o menos una vez al día) fre- \\
& cuencia respiratoria: >25 resp/min (taquipnea) con saturacio- \\
& nes de oxígeno bajas: $81 \%$. Por lo cual utiliza gafas nasales \\
& a 2 L y respiración ineficaz y superficial, utilizando la muscula- \\
& tura accesoria para realizar los movimientos respiratorios (es- \\
& piración e inspiración) y el paciente refiere dolor agudo inter- \\
& mitente en la zona de incisión quirúrgica y drenajes en dichos \\
& episodios. \\
& - Presenta dos drenajes torácicos derechos; uno anterior y el \\
& otro posterior, ambos permeables. El anterior; a nivel de la \\
& línea medio clavicular, y el posterior a la línea axilar. Ambos \\
& utilizados para drenar el derrame pleural post-cirugía (lobecto- \\
& mía inferior derecha más linfadenectomía). \\
\hline
\end{tabular}




\begin{tabular}{|c|c|}
\hline 2. Necesidad de eliminación & $\begin{array}{l}\text { Drenajes torácicos derechos permeables: } \\
\text { - Anterior (línea medio clavicular derecha): Drenaje sin fugas } \\
\text { aéreas y en pasiva. Cantidad adecuada: } 1^{\text {er }} \text { día: } 500 \mathrm{cc} / 24 \mathrm{~h} \text { con } \\
\text { contenido serohemático. } 2^{\circ} \text { día: } 350 \mathrm{cc} \text { de contenido serohe- } \\
\text { mático. } \\
\text { - Posterior (línea axilar): Drenaje sin fugas aéreas y en pasiva. } \\
\text { Cantidad adecuada } 1^{\text {er }} \text { día postoperatorio; } 200 \mathrm{cc} \text { serohemáti- } \\
\text { co. } 2^{\circ} \text { día: } 100 \mathrm{cc} \text { con contenido hemático. } 3^{\text {er }} \text { día: } 50 \mathrm{cc} \text { sero- } \\
\text { hemático. } \\
\text { - Ayuda parcial y supervisión para desplazarse al inodoro, du- } \\
\text { rante los tres días posoperatorio, debido a la presencia del } \\
\text { catéter venoso periférico colocado en la mano derecha con } \\
\text { suero en perfusión, por la presencia de los drenajes torácicos } \\
\text { y el dolor en la región local de inserción de los mismos, ade- } \\
\text { más del dolor lateral-posterior-superior costal donde se sitúa la } \\
\text { herida quirúrgica. }\end{array}$ \\
\hline $\begin{array}{l}\text { 3. Necesidad de nutrición } \\
\text { e hidratación }\end{array}$ & $\begin{array}{l}\text { - Durante los } 3 \text { días postoperatorio mantiene catéter venoso } \\
\text { periférico con suero en perfusión: } 1^{\text {er }} \text { día: } 400 \mathrm{ml} / 24 \mathrm{~h} \text { de suero. } \\
2^{\circ} \text { día: } 300 \mathrm{ml} / 24 \mathrm{~h} \text { de suero, } 3^{\text {er }} \text { día: } 300 \mathrm{ml} / 24 \mathrm{~h} \text { de suero }\end{array}$ \\
\hline $\begin{array}{l}\text { 4. Necesidad de moverse } \\
\text { y mantener buena postura }\end{array}$ & $\begin{array}{l}\text { Movimiento: } \\
\text { - Dificultad moderada para moverse: levantarse, sentarse, ca- } \\
\text { minar. Debido al dolor agudo intermitente en la región torácica } \\
\text { y costal derecha, concretamente en la región local de inser- } \\
\text { ción de drenajes y en la zona de la herida quirúrgica, durante } \\
\text { los tres días del posoperatorio. Evaluado el dolor mediante la } \\
\text { escala numérica, el paciente refiere número } 8 \text {. La puntuación } \\
\text { corresponde a calificar el dolor como intenso. Otra causa por } \\
\text { la cual se dificulta el movimiento, en concreto el desplaza- } \\
\text { miento, es la presencia de los drenajes y el sistema de suero. } \\
\text { Dependiente en grado moderado en el desplazamiento. Ha } \\
\text { sido evaluado el riesgo de caídas mediante la escala de Morse } \\
\text { y ha obtenido una puntuación de } 45 \text {, lo que se traduce por } \\
\text { riesgo moderado para movilidad. } \\
\text { Nivel hematológico: } \\
\text { - Debido a la cirugía y a la merma en su movilidad, el paciente } \\
\text { presenta riesgo de trombosis venosa profunda, por tanto, se le } \\
\text { administra todos los días a las } 9 \mathrm{~h} \text { enoxaparina sódica, bajo } \\
\text { prescripción médica. }\end{array}$ \\
\hline
\end{tabular}




\begin{tabular}{|c|c|}
\hline $\begin{array}{l}\text { 5. Necesidad de evitar } \\
\text { peligros }\end{array}$ & $\begin{array}{l}\text { - Presencia de dolor agudo intermitente en la región derecha } \\
\text { lateral-posterior-superior costal donde está la herida quirúrgi- } \\
\text { ca, donde tuvo lugar la lobectomía y la linfadenectomía, y la } \\
\text { inserción de los drenajes torácicos en la región derecha (línea } \\
\text { medio clavicular y línea axilar). Este dolor es caracterizado } \\
\text { como intenso, por la valoración mediante la escala numérica, } \\
\text { la cual dio una puntuación de } 8 \text {. } \\
\text { - Predisposición a los accidentes: riesgo de caída moderado ya } \\
\text { que se obtuvo una puntuación de } 45 \text {. Valorado mediante la } \\
\text { escala de Morse. Debido a: disminución en la marcha y la mo- } \\
\text { vilización (por causa de la presencia del dolor agudo intermi- } \\
\text { tente intenso en el sitio de la intervención e inserción de dre- } \\
\text { najes, por la presencia de dichos drenajes y por el sistema de } \\
\text { suero). } \\
\text { - Predisposición a la inflamación e infección: por la presencia } \\
\text { del catéter venoso periférico en perfusión en la mano derecha, } \\
\text { y la presencia de los drenos torácicos derechos inseridos en el } \\
\text { espacio pleural; anterior (línea medioclavicular) y posterior } \\
\text { (línea media axilar). } \\
\text { - Control del patrón respiratorio para evitar una insuficiencia } \\
\text { respiratoria; controlando las Sat } 0_{2} \text {, FR, y controlando el PaO }{ }_{2} \text {, } \\
\text { Paco }{ }_{2} \text {, pH mediante la realización de las gasometrías. }\end{array}$ \\
\hline $\begin{array}{l}\text { 6. Necesidad de estar } \\
\text { limpio, aseado y proteger } \\
\text { sus tegumentos }\end{array}$ & $\begin{array}{l}\text { Aseo: } \\
\text { - Precisa de ayuda parcial en la higiene corporal: ayuda parcial } \\
\text { en la transferencia hasta el baño y ayuda en la higiene de las } \\
\text { piernas, pies y zona perianal. } \\
\text { Integridad piel: } \\
\text { - Presencia de herida quirúrgica, en la zona lateral-posterior- } \\
\text { superior costal derecha con suturas internas, buen aspecto, } \\
\text { cicatrizante por primera intención y con ausencia de signos } \\
\text { inflamatorios (rubor, calor e coloración enrojecida), sin apósito } \\
\text { y ausencia de hemorragia, debido a la intervención quirúrgica } \\
\text { de lobectomía y linfadenectomía. Zona de inserción de los } \\
\text { drenajes torácicos anterior (línea media clavicular) y posterior } \\
\text { (línea axilar) y catéter venoso periférico en la mano derecha, } \\
\text { buen aspecto y con ausencia de signos inflamatorios. Ambos } \\
\text { están cubiertos con un apósito. }\end{array}$ \\
\hline $\begin{array}{l}\text { 7. Mantener la temperatura } \\
\text { corporal }\end{array}$ & No presenta manifestaciones de dependencia. \\
\hline 8. Vestirse y desvestirse & $\begin{array}{l}\text { Precisa ayuda parcial para vestirse y desvestirse en concreto } \\
\text { con los pantalones y los zapatos, durante los tres días pos } \\
\text { operatorio debido a la movilidad reducida y viste el pijama del } \\
\text { hospital. }\end{array}$ \\
\hline 9. Dormir y descansar & No presenta alteraciones de dependencia. \\
\hline $\begin{array}{l}\text { 10. Ocuparse para } \\
\text { realizarse }\end{array}$ & Muestra baja autoestima y se muestra poco asertivo. \\
\hline
\end{tabular}




\begin{tabular}{|l|l|}
\hline 11. Comunicar & No presenta alteraciones de dependencia. \\
\hline $\begin{array}{c}\text { 12. Actuar según sus } \\
\text { creencias y valores }\end{array}$ & No presenta alteraciones de dependencia. \\
\hline 13. Aprender & No presenta alteraciones de dependencia. \\
\hline 14. Recrearse & No presenta alteraciones de dependencia. \\
\hline
\end{tabular}

